\title{
ON SECOND-ORDER FUNCTIONAL DIFFERENTIAL INCLUSIONS IN HILBERT SPACES
}

\author{
Myelkebir Aitalioubrahim
}

Abstract. We prove the existence result of monotone solutions, in Hilbert space, for the differential inclusion $\ddot{x}(t) \in f(t, T(t) x, \dot{x}(t))+F(T(t) x, \dot{x}(t))$, where $f$ is a Carathéodory single-valued mapping and $F$ is an upper semicontinuous set-valued mapping with compact values contained in the Clarke subdifferential $\partial_{c} V(x)$ of a uniformly regular function $V$.

Mathematics subject classification (2010): 34A60, 34K05, 34K25.

Keywords and phrases: functional differential inclusions, regularity, Clarke subdifferential.

\section{REFERENCES}

[1] M. Aitalioubrahim, S. Sajid, Second-order viability problem: A Baire category approach, Bull. Pol. Acad. Sci. Math., 57 (2009), 9-23.

[2] M. Aitalioubrahim, S. Sajid, Second-order viability result in Banach spaces, Discuss. Math. Differ. Incl. Control Optim., 30 (2010), 5-21.

[3] J. P. Aubain, A. Cellina, Differential inclusions, Springer-Verlag Berlin, 1984.

[4] A. Auslender, J. MeChler, Second-order viability problems for differential inclusions, Academic Press Inc., 1994.

[5] M. BounKHEL, Existence results of nonconvex differential inclusions, Port. Math., 59, 3 (2002), 283310 .

[6] A. CERnEA, On the existence of viable solutions for a class of second-order differential inclusions, Discuss. Math., Diff. Incl. Control Optim., 22 (2002), 67-78.

[7] F. H. Clarke, Optimization and nonsmooth analysis, Wiley and Sons, 1983.

[8] F. H. Clarke, YU. S. Ledyaev, R. J. Stern, P. R. Wolens Ki, Nonsmooth analysis and control theory, Springer New York, 1998.

[9] A. Gavioli, L. Malaguti, Viable solutions of differential inclusions with memory in Banach spaces, Port. Math., 57, 2 (2000).

[10] G. HADDAD, Monotone trajectories of differential inclusions and functional differential inclusions with memory, Israel J. Math., 39 (1981), 83-100.

[11] G. HAdDAD, Monotone trajectories for functional differential inclusions, J. Differential Equations, 42 (1981), 1-24.

[12] A. G. Ibrahim, F. A. AL-AdSAni, Monotone solutions for a nonconvex functional differential inclusions of second-order, Electron. J. Differential Equations, 144 (2008), 1-16.

[13] V. LUPULESCU, Viable solutions for second-order nonconvex functional differential inclusions, Electron. J. Differential Equations, 110 (2005), 1-11.

[14] V. LupUlESCU, A viability result for second-order differential inclusions, Electron. J. Differential Equations, 76 (2003), 1-12.

[15] V. LuPUlESCU, Existence of solutions for nonconvex second-order differential inclusions, Appl. Math. E-Notes, 3 (2003), 115-123.

[16] R. T. RockAFELlaR, Generalized directional derivatives and subgradients of nonconvex functions, Canad. J. Math., 39 (1980), 257-280.

[17] A. Syam, Contributions aux inclusions différentielles, Doctoral thesis, Université Montpellier II, 1993. 